\title{
Benefits of antioxidant supplements for knee osteoarthritis: rationale and reality
}

\author{
Ashok Kumar Grover ${ }^{*}$ (D) and Sue E. Samson
}

\begin{abstract}
Arthritis causes disability due to pain and inflammation in joints. There are many forms of arthritis, one of which is osteoarthritis whose prevalence increases with age. It occurs in various joints including hip, knee and hand with knee osteoarthritis being more prevalent. There is no cure for it. The management strategies include exercise, glucosamine plus chondroitin sulfate and NSAIDs. In vitro and animal studies provide a rationale for the use of antioxidant supplements for its management. This review assesses the reality of the benefits of antioxidant supplements in the management of knee osteoarthritis. Several difficulties were encountered in examining this issue: poorly conducted studies, a lack of uniformity in disease definition and diagnosis, and muddling of conclusions from attempts to isolate the efficacious molecules. The antioxidant supplements with most evidence for benefit for pain relief and function in knee osteoarthritis were based on curcumin and avocado-soya bean unsaponifiables. Boswellia and some herbs used in Ayurvedic and Chinese medicine may also be useful. The benefits of cuisines with the appropriate antioxidants should be assessed because they may be more economical and easier to incorporate into the lifestyle.
\end{abstract}

Keywords: Arthritis, Turmeric, Curcumin, Piperine, Avocado, Boswellia, Superoxide, Peroxide, Peroxynitrite

\section{Current knowledge}

Arthritis may have originated before man itself since it also afflicts other primates [1]. The disease causes disability due to pain and inflammation in joints. There are many different types of arthritis of which rheumatoid arthritis and osteoarthritis (OA) are the most common. Rheumatoid arthritis is an autoimmune disease that affects mainly small joints such as those in the fingers of the hand. OA affects large joints such as hips and knees and also those in the hands. OA is a leading cause of disability with an unknown cause or cure. The global age standardised prevalence of OA in the knee and hip has recently been reported to be 3.8 and $0.85 \%$, respectively [2]. Worldwide estimates indicate that $9.6 \%$ of men and $18 \%$ of women $\geq 60$ years have symptomatic OA (http://www.who.int/chp/topics/rheumatic/en/). Other risk factors of knee OA include trauma such as torn meniscus, occupation, exercise, gender (more common in females), ethnicity, genetics, obesity, diet and bone density [2]. Since OA normally progresses with

* Correspondence: groverak@mcmaster.ca

Department of Medicine, HSC 4N41, McMaster University, 1280 Main Street W., Hamilton, ON L8S 4K1, Canada age, its economic burden may increase with the aging human population in the coming decades. This review will focus on knee OA which is more common. The knee joints are the largest and being synovial joints, they provide a very high degree of mobility. A knee joint provides two articulations - one between tibia and femur and the other between patella and femur $[3,4]$. The joints allow for flexion, extension and a limited degree of rotation. It contains a bone-cartilage interface and a synovial body. The synovial body contains the fluid whose composition and viscosity are key to the knee operation. The bone-cartilage interface is a complex functional unit and biocomposite at the centre of joint function in which the individual components interact cooperatively and synergistically. Due to this intimate contact between bone and cartilage, any changes in either tissue will influence the other component. Its role in knee $\mathrm{OA}$ is discussed in the next section.

\section{Pathophysiology of knee OA}

The suggested causes for the development of OA include genetic predisposition, aging, obesity, trauma, and 
other systemic diseases [5]. Irrespective of the etiology, a number of processes that occur in the initial stages may involve cellular and ultrastructural changes which gradually accompany the phenotypic image of OA. During OA there is a loss of cartilage, the subchondral bone becomes thicker, the subchondral trabecular bone mass decreases and new osteophytes are formed [3]. These changes may lead to the development of bone cysts and lesions in the bone marrow. Subsequently, the cartilage layer may be calficified and cracks may occur in it. The chondrocytes, which are normally quiescent, may also actively proliferate and form clusters. An early-stage increase in the remodelling and bone loss followed by a slow remodelling and subchondral densification are hallmarks of OA pathogenesis [6].

It is commonly stated that the articular cartilage protects the bone to prevent any damage during motion, however, the articular cartilage, subchondral plate and trabecular bone are a biologically and functionally inseparable osteochondral unit which absorbs and distributes loads across the joint $[7,8]$. The osteochondral plate consists of the following: a thin layer of cortical bone (also called the subchondral plate), a calcified layer of cartilage, adjacent articular cartilage and the tidemark. The subchondral plate is a highly vascular cortical bone. The entire osteochondral plate should be viewed as an exchange area between bone and cartilage through which the bone supplies the cartilage with nutrients and oxygen. Due to this intimate contact between bone and cartilage, any changes in either tissue will influence the other component. The distance between the two bones is important because a decrease in this distance may increase friction during motion. The muscles and the joint are vascularized and innervated. Thus, changes in the elements in the supplied blood may also influence the properties of the joint. This also means that signals from the nerves may lead to a neuropathic pain in the joint [3].

During OA there is a loss of cartilage, the subchondral bone becomes thicker, the subchondral trabecular bone mass decreases and new osteophytes are formed [3]. It has been shown that the subchondral bone sclerosis is characterized by a trabecular thickening and a decrease in calcium binding to the collagen fibers. This abnormal mineralization is due to the overproduction of the homotrimeric alpha1 form of type I collagen by osteoblasts. This $(\alpha 1) 3$ type I collagen has a lower affinity for calcium than the $(\alpha 1) 2 \alpha 2$ type I collagen [7, 8]. This is consistent with a recent study using Raman spectroscopy which concluded that the chemical compositions and collagen quality were different between subchondral bone in the advanced OA and the non-OA distal femur [9]. There may also be changes in the parathyroid hormone induced cAMP levels, and vitamin D induced production of alkaline phosphatase and osteoclacin [7]. OA osteoblasts produced more insulin-like growth factor-1 and urokinase than normal cells. Other changes in the subchondral osteoblasts in OA may be altered production of interleukin (IL)-6, IL-8, metalloproteases and transforming growth factor (TGF)- $\beta 1$. Increased Wnt signaling in the subchondral bone can also contribute to OA development [7]. It is reminded that there is a close cross-talk between the various tissues in the osteochondral unit and hence changes in the subchondral bone may affect the cartilage and the reverse may also occur. The precise locale of the changes in OA within this unit remains to be established. These changes may lead to the development of bone cysts and lesions in the bone marrow. Subsequently, the cartilage layer may be calficified and cracks may occur in it. The chondrocytes, which are normally quiescent, may also actively proliferate and form clusters. An early-stage increase in the remodelling and bone loss followed by a slow remodelling and subchondral densification are hallmarks of OA pathogenesis [6].

OA affects the entire joint: the cartilage is damaged, the underlying subchondral bone structure is remodelled, and a chronic inflammation of the synovium develops [4]. There is sufficient evidence that during OA the cross-talk between different tissues in the joint becomes more pronounced $[3,7,8]$.

A current thinking is that $\mathrm{OA}$ is a chronic inflammation disease that occurs with gradual changes in the immune system. This hypothesis has been presented in detail in a recent review [5]. The progression of OA involves changes in the production and functioning of various cytokines. The cytokines involved may be inflammatory interleukins (IL-1 $\beta$, IL-6, IL-15, IL-17, and IL-18) and tumour necrosis factor-alpha (TNF- $\alpha$ ) or anti-inflammatory interleukins (IL-4, IL-10,and IL-13). The increase in IL-1 causes damage to the articular cartilage [5]. The effect of TNF- $\alpha$ is similar to and synergistic with the actions of IL- $1 \beta$. The net result is a blockage of the synthesis of proteoglycan components, the proteins which bind proteoglycans, and type II collagen in chondrocytes $[10,11]$. Activated chondrocytes also produce the matrix metalloproteases MMP-1, MMP3,MMP-13 [5]. The effect of IL-6 on the cartilage is similar to and in synergy with that of the other inflammatory cytokines and leads to a decrease in the synthesis of type II collagen and an increase in the matrix metalloprotease activity $[5,12]$. Serum concentrations of IL-5 have been associated with the severity of pain in OA [13]. However, the concentrations in the synovial fluid are higher in early knee OA patients when compared to end-stage OA [14]. The level of IL-17 increases in the serum and in the synovial fluid. This level in the latter correlates positively with the radiographic image of lesions in OA [15]. IL-18 affects chondrocytes and 
synovial cells by increasing the levels of several inflammatory compounds [5]. The anti-inflammatory cytokines act primarily by decreasing the levels of inflammatory cytokines, especially IL- $1 \beta$ and TNF- $\alpha$ [5]. Thus, there is sufficient evidence to support the immune hypothesis in OA.

\section{Diagnosis of knee OA}

Assessment of efficacy of a therapy requires an understanding of diagnosis of a disease such as OA which is first reported by the patient as pain and inability to perform certain routine physical tasks. The clinician diagnoses it as OA by ruling out other causes such as rheumatoid, psoriatic and septic arthritis, injury and other causes.

The diagnosis of the severity of $\mathrm{OA}$ is subjective: based on a quality of life questionnaire, physical examination and radiography (for a summary see [4]). The most commonly used quality of life measure is Western Ontario McMaster Index (WOMAC) although other similar measures such as visual analog scale (VAS) for pain and Lequesne index are also used [16, 17]. An example of the WOMAC questionnaire for the knee is at http:// www.orthopaedicscore.com/scorepages/knee_injury_osteopaedic_outcome_score_womac.html. The WOMAC questionnaire contains several parts and contains questions related to severity and frequency of symptoms such as swelling of the joint, grinding and clicking noises, knee catching or hanging up, and the ability to straighten or bend knees, pain in the knees in different positions, knee functions and ability to perform daily functions. Based on the sum of all the scores, the overall WOMAC score is determined. Higher scores indicate greater severity.

The physical exam focuses on the range of motion (both passive and active), muscle strength, ligament stability and tenderness of the affected joints [4]. Any swelling due to inflammation is also examined. Examination by palpation of the OA knee may show the presence of a crepitus (a crackling or crunching sensation on range of motion of the joint).

Diagnosis of OA by imaging techniques has recently been summarised [18]. Traditionally it has been diagnosed with X-ray radiographs from different angles to look for joint space width (JSW) and asymmetry, and for the presence of osteophytes. Grading schemes to describe the severity of the disease based on radiography have been developed but these are questioned by others since this method does not yield much information on changes in soft tissue and cartilage [19]. Therefore, the more recent methods such as magnetic resonance imaging (MRI), ultrasound, and optical coherence tomography, have enhanced the OA diagnosis [18]. However, most clinical trials have not used these methods due to their high cost, low availability and the unavailability of literature establishing their association with other criteria of osteoarthritis. In most trials the assessment of pain and function has been used.

\section{Current therapies for management of knee OA}

There are a large number of treatments of varying efficacy and faith for OA. The non-pharmacological treatments include education, exercise, physiotherapy, weight loss, physical aids (supports, braces and walkers) and surgical joint replacements [16, 20-24]. Massage with and without pharmacological agents may also be beneficial. Vitamins and herbs have also been used. A number of recommendations on the treatments for OA made by the American Academy of Orthopaedic Surgeons (AAOS) are available at the website http://www.aaos.org/Research/guidelines/GuidelineOAKnee.asp.

Physical aids may enable the mobility of the patients and allow them to carry out more physical activity. Thus, even then the aim remains an increased physical activity through some form of exercise. Weight loss is the common mantra of the health care professionals for the management of $\mathrm{OA}$, it is moderately recommended by AAOS but its validity may be questionable [25]. A variety of exercise programs of varying effectiveness are available for knee arthritis. It appears that for OA patients, exercises involving supervised slow movements or isometric exercises may be efficacious and also have a lower possibility of damage to the joint than other exercises $[16,20-23,26]$. Therefore, aquatic exercises, yoga and tai chi should be preferred. This is one of the strongest recommendations from the AAOS. Running on treadmills should be avoided. For less severe OA some exercises with slower movements and greater resistance may be added. Since the type, intensity and dose of exercise may benefit each patient differently, physiotherapy is often used to determine the type and extent of the exercises [23]. It is claimed that primary care physiotherapists and pharmacists may improve short term outcomes for older adults with knee pain and reduce the use of NSAIDs [26]. A long term randomised trial which will monitor the effectiveness of community therapy and enhanced pharmacy review for people over the age of 55 has been initiated [20]. It may better resolve these claims.

Several pharmacological agents have been used for management of OA. Temporary pain relief and hence improvement in function may be obtained with analgesics but this is not specific to OA. NSAIDs are used orally and topically because they have some antiinflammatory and analgesic effects. They are also strongly recommended by AAOS. However, they may have severe adverse effects upon prolonged use. These issues have been discussed in recent reviews and will not 
be retraced here $[27,28]$. There are several commercial preparations such Instaflex, Sierrasil, hyaluronic acid and Aquamin of limited proven usefulness [29-31]. However, the combination of glucosamine and chondroitin sulfate is the most promising. This treatment may be efficacious for pain relief, functional improvement and also result in less joint space narrowing [32-36].

Herbs have been used for such treatment since ancient times in Indian medicine (Ayurvedic) and Chinese medicine $[37,38]$. The use of some herbs is also mentioned in the Bible (http://www.threemagi.com/frankincense.html). Most of these herbs have antioxidant properties: they contain compounds or chemicals that can modulate oxidative metabolism which is altered during OA. Many in vitro studies are available in this area. However, the human body is more complex than the cells cultured in defined growth media. Oxidative stress may play a role in several diseases but the benefits of different antioxidant supplements may be unique to each one. We recently reviewed the literature on the benefits of antioxidants in vision health and in obesity-diabetes II $[39,40]$. In vision health, the antioxidant supplements containing vitamin $\mathrm{C}$, vitamin $\mathrm{E}$, lutein, zeaxanthin, zinc and copper have a reasonable probability of retarding age-related macular degeneration but the benefits in other eye diseases are questionable. In obesity and diabetes 2 , there are marginal benefits of supplementation with zinc, lipoic acid, carnitine, cinnamon, green tea, and possibly vitamin $\mathrm{C}$ plus E. Some of the antioxidants are beneficial for obesity and others are better for glucose level regulation.

\section{Antioxidant supplements in knee $O A$ Reactive oxygen species in $\mathrm{OA}$}

A free radical is a molecule with an unpaired electron in its outermost orbit $[41,42]$. In biological systems, a free radical that involves oxygen is termed a reactive oxygen species (ROS) but the term ROS is used loosely for oxidants such as peroxides. Normal physiological processes result in the generation of ROS such as peroxide, superoxide, hydroxyl radical and peroxynitrite [41, 42]. Thus, ROS occur normally in the body at very low concentrations (nanomolar to micromolar). They are a necessary evil since our body needs them for survival but, when in excess, they may have deleterious effects. Our body gets rid of the excess ROS using natural antioxidants such as vitamin $\mathrm{C}$ (ascorbate), vitamin $\mathrm{E}$, glutathione and various enzymes [41-43]. The term oxidative stress is used as a measure of the overall ROS status. It is the ratio of the amount of peroxide present to that of the antioxidant capacity of the cell. High levels of oxidative stress may damage the cells by oxidising lipids and by altering DNA and protein structure.
The concentrations of different ROS, reduced and oxidised thiols, oxidative stress index and the related enzymes have been monitored in OA. In one study, the serum thiol levels and catalase activity were lower in advanced stage $\mathrm{OA}$ patients than in controls and the oxidative stress index was also higher $(P<0.001$, for all $)$ [44] (Fig. 1). In this work the levels of prolidase (a cytosolic exopeptidase which cleaves imidodipeptides and imidotripeptides with C-terminal proline or hydroxyproline) activity correlated negatively with the oxidative stress index. Another study examined the synovial fluid from OA patients undergoing total knee replacement surgery [45]. In these patients, the lipid peroxidation and antioxidants were similar to those with the injured knee joint patients but Vitamin E deficiency was associated with OA [45]. However, a similar study found that the extracellular superoxide dismutase (SOD) levels were lower in the advanced stage OA patients than with the knee injury controls [46]. There was also decline in the concentrations of reduced glutathione and ascorbate [46].

\section{The above paradigm of oxidative stress is too simplistic}

The kinetic constraints indicate that in vivo scavenging of ROS is ineffective as an antioxidant defense [43, 47]. The concept does not consider that the damage may be unique to each ROS in different cell types. Also, often individual ROS species may act as signals. Therefore, a better concept of oxidative stress is that of a disruption of redox signalling and control [48]. In discussing knee OA, this would be the effects on synovium, cartilage and the joints. These effects relate directly to the OA pathophysiology. For example, IL- $1 \beta$ is one of the most active cytokines during the development of $\mathrm{OA}$ and it stimulates the production of ROS such as peroxides and hydroxylated radicals and the production of $\mathrm{NO}$ and a deficiency in SOD. The deficiency in SOD leads to higher levels of superoxide. $\mathrm{NO}$ and superoxide react to form peroxynitrite [46] (Fig. 1). Peroxynitrite can cause telomere erosion by targeting guanine repeats in the DNA. The net result is a decrease in the synthesis of collagen II which is required for the maintenance of the cartilage. Another potential pathway by which ROS can damage the joint is through lipid peroxidation which produces 4-hydroxynonenal. Higher levels of 4hydroxynonenal are present in the synovial cells of OA compared to those of the healthy subjects [49]. In cartilage explants, 4-hydroxynonenal induced cleavage of collagen II [49]. In chondrocytes isolated from OA patients, 4-hydroxynonenal inhibited the expression of collagen II and increased the levels of factors which can cause its degradation [49]. Thus, the production of 4-hydroxynonenal by ROS could play a major role in OA (Fig. 1). It is reminded that the joint is a system in which the cartilage, 


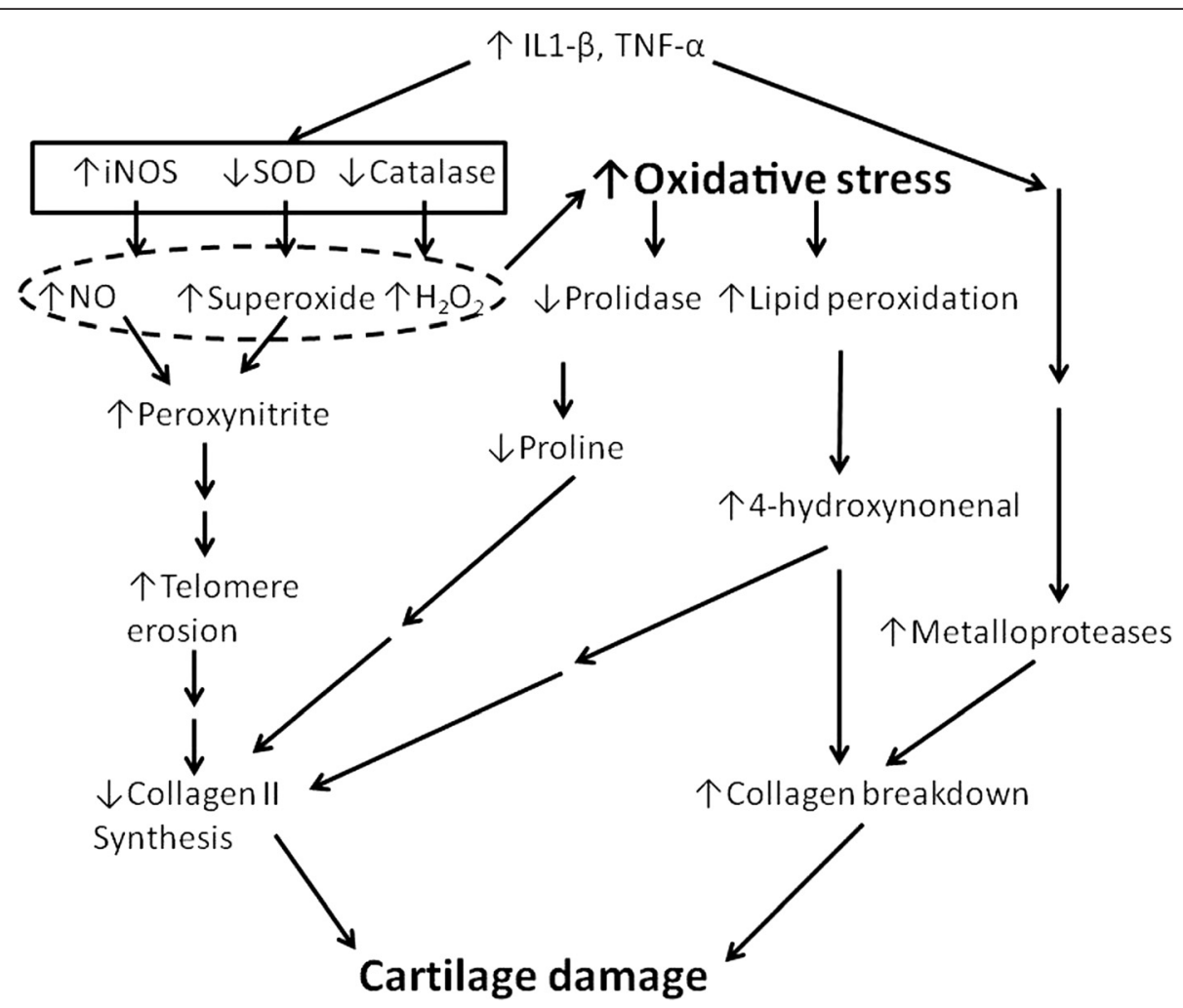

Fig. 1 Role of oxidative stress in cartilage damage during OA. OA is hypothesised as a chronic inflammation disease that occurs with gradual changes in the immune system (see Pathophysiology of knee OA). IL-1 $\beta$ and TNF- $a$ and other inflammatory factors increase in OA. This pathway leads to induction of NO synthase, production of larger amounts of NO and a deficiency in SOD and catalase (see Role of ROS in OA). The deficiency in SOD leads to higher levels of superoxide which combines with NO to produce peroxynitrite which can cause telomere erosion by targeting guanine repeats in their DNA telomeres. The net result is a decrease in the synthesis of collagen II. The decrease in catalase results in accumulation of peroxide to increase lipid peroxidation which produces 4-hydroxynonenal. The 4-hydroxynonenal increases factors which breakdown collagen II and also inhibits the expression of collagen II. The net result is the cartilage damage that occurs in OA. Note that the scheme shown here is only a summary

the bone, the ligaments and the synovium form a capsule and there is sufficient cross-talk between all the tissues. A diffusion of ROS and lipid peroxidation products may occur between them. Thus, the damage to one element of the joint may influence others through fluid diffusion and by paracrine factors [50].

\section{Antioxidant supplements and $\mathrm{OA}$}

The role of ROS in the pathophysiology of knee OA provides for the rationale that suppressing the ROS levels with the appropriate antioxidant supplements may retard the progress of the disease. What remains to be discussed is the reality of the observations on the effects of such supplements on prevention and/or management of OA.

The effects of food intake and various vitamins and related compounds on $\mathrm{OA}$ has been examined and reviewed [51-59]. One concludes that nutritional habits involving fruits, fruit juices and vitamin supplements may be beneficial in the long run but they may not help once OA has already been initiated. Several antioxidant supplements derived from turmeric, avocado, Boswellia and other herbs will be discussed.

\section{Turmeric}

Turmeric is used extensively as a spice and has been used for over 4000 years as an Ayurvedic medicine. It is the rhizome of Curcuma longa (some studies use Curcuma domestica) and contains over 20 different active compounds [60]. Curcumin, a compound with antioxidant properties, was isolated from turmeric about 200 years ago [61]. The properties of curcumin and its potential role in the therapy of several chronic diseases including arthritis, cancer and neuronal disorders have been explored. The rationale for its use in OA comes from several in vitro studies. Curcumin inhibited the matrix degradation of articular explants and chondrocytes [51]. It decreased the production of MMP-3, -9 and -13 via c-Jun-N-terminal kinases, nuclear factor 
kappa-beta $(\mathrm{NF} \kappa \beta)$, and the JAK/STAT pathways. It also restored type II collagen and glycosaminoglycan synthesis. A random double blind study on knee OA patients compared the ability of curcuminoids and the NSAID diclofenac to inhibit cyclo-oxygenase 2 [62]. Both the groups significantly reduced $(p<0.001)$ cyclo-oxygenase 2 secretions by similar efficacies.

A PubMed search in August 2015 with the words "tumeric/curcumin" and "osteoarthritis" and "knee" showed 21 entries of which 11 were clinical trials involving pain and function with different diagnostic measurements, durations, controls, blindness and conflicts of interests (Table 1). All the trials showed benefits of curcumin except for one in which significance levels were not attained due to a large variance. Some studies have also compared their effects with those of NSAIDs. One randomised double blind study compared the effects of ibuprofen $(2 \times 400 \mathrm{mg} /$ day $)$ with those of curcumin $(4 \times$ $500 \mathrm{mg} /$ day) in patients who were over 50 years of age, had severe knee pain and their radiography showed the presence of osteophytes [63]. Both the groups showed improvements in all assessments but the curcumin group was statistically better in patient satisfaction, timed walk or stair climbing and pain during walking or stair climbing. A similar study with a larger number of patients compared the effects of $C$. domestica extracts $(1500 \mathrm{mg} /$ day, $n=171)$ with ibuprofen $(1200 \mathrm{mg} /$ day $n$ $=160$ ) for 4 weeks [64]. After 4 weeks, all measures of WOMAC and the 6 min walk test improved significantly $(p<0.001)$ in both groups with no differences between the groups. The safety profile was found to be somewhat better in the curcumin group. Another study compared the effects of the NSAID diclofenac $(75 \mathrm{mg}$ ) combined with placebo $(n=36)$ or with Curcuma longa extracts (1000 mg) $(n=37)$ on pain and function. The combination was no more effective than diclofenac alone indicating that the effects of the two treatments are not additive (Table 1) [65].

A major criticism is that most studies did not report curcumin levels in the patients. This is important because curcumin absorption has been reported to be extremely poor when it is used alone [66-69]. To further complicate matters, one study reports that even a curcuminoid free polysaccharide rich extract (NR-INF-02) of Curcuma longa may be efficacious against OA (Table 1) [70].

There have been several attempts to improve the bioavailability of curcumin. One study examined the effects of Meriva (curcumin plus phosphatidylcholine for better bioavailability) (Table 1) [71]. Patients $(n=100)$ with mild OA were recruited. They were already under various treatments. The control group continued with only the current treatment but Meriva $(2 \times 500 \mathrm{mg} /$ day-totalling $200 \mathrm{mg}$ curcumin) was added to the study group.
After 8 months, the control group showed an improvement on the WOMAC scale but the treatment group performed much better. The mean treadmill test distances changed from 82.3 to $156 \mathrm{~m} / 6 \mathrm{~min}$ in the control group but the increase was much greater in the treatment group (from 77.3 to $344 \mathrm{~m} / 6 \mathrm{~min}$ ). All the inflammatory markers (IL6, IL1 $\beta$, sVCAM-1, sCD40L, ESR) were significantly decreased $(p<0.05)$ in the study group but not in the control group. Another study reported the effects of Meriva and glucosamine $(n=63)$ vs chondroitin and glucosamine $(n=61)$ in patients with mild OA [66]. The patients improved more with Meriva plus glucosamine than with chondroitin plus glucosamine in WOMAC scores and in the treadmill test. No adverse effects were reported. However, this was not a randomised blind study. It is pointed out that the levels of curcumin in the patients were not reported in either study using Meriva [66, 71]. Another study mixed curcumin with polysorbate for better bioavailability [67]. Curcumin (42 $\mathrm{mg} /$ capsule, $2 \times 3 /$ day) was given for 3 months. The treatment decreased collagen II but had no significant effects on pain or other biomarkers measured. In another multicenter larger study this combination was also beneficial for pain and flexibility [72]. However, curcumin levels in the patients were not reported in either study. An intravenous infusion of high concentrations of liposomes containing curcumin altered the shape of erythrocytes [73].

One study determined the effect of piperine (inhibits hepatic and intestinal glucuronidation) on the bioavailability of curcumin [69]. After a dose of $2 \mathrm{~g}$ curcumin alone, the curcumin levels in the serum were near or below the detection limit. In contrast, a coadministration of $20 \mathrm{mg}$ piperine increased the bioavailability by 20 -fold $(p<0.01)$. It was concluded that piperine enhanced the serum concentration, extent of absorption and bioavailability of curcumin with no adverse effects. A pilot random double blind study with 53 patients was conducted on the effects of curcumin plus bioperine $(3 \times(300 \mathrm{mg}$ curcumin $+5 \mathrm{mg}$ piperine)/day) or a placebo for 6 weeks (Table 1) [68]. The treatment led to significant improvements in pain relief and function compared to the placebo. However, there are three concerns with this study. There was no control group without piperine, curcumin levels were not determined, and there was no control group with piperine alone. Therefore, the results are difficult to interpret. An in vitro study on chondrocytes from an OA patient showed that piperine alone can abrogate the IL1-beta-induced overexpression of inflammatory mediators [74].

Curcumin has been reconstituted with noncurcuminoid components of turmeric into a proprietary preparation termed BCM-95CG or Biocurcumax [75]. 
Table 1 Knee OA antioxidant supplements based on turmeric, avocado and Boswellia

Turmeric (curcumin)

Kuptniratsaikul RDBa: compared curcumin $(n=171)$ et al. 2014 [63, with ibuprofen $(n=160)$

64]

Belcaro et al. Open: best available treatment + $2010[71]$ Meriva versus best available treatment $(n=100)$

Belcaro et al. Open: Meriva and glucosamine $(n=$ in 2014 [66] 63) vs chondroitin and glucosamine $(n=61)$

Panahi et al. in RDBP: curcumin + bioperine $(n=21)$ vs WOMAC, VAS, Lequesne's pain and $2014[66,68] \quad$ placebo $(n=19)$

Pinsornsak DB: (Curcumin $1000 \mathrm{mg}+$ diclofenac 2012 [65] $\quad 75 \mathrm{mg}) /$ day $(n=44)$ vs (diclofenac

$75 \mathrm{mg}+$ placebo) $(n=44)$

Henrotin, $Y$ et Open: Flexofytol (curcumin with al. 2014 [67] polysorbate) $(n=100)$ in real life situations

Appelboom et Open: Flexofytol- physicians in real life al. 2014 [72] situation $(n=820)$

Kertia 2012 RDB: C. domestica $3 \times 30 \mathrm{mg}(n=34)$ [62] vs diclofenac $3 \times 25 \mathrm{mg}(n=39)$

Madhu 2013 RSBP: NR-INF-02 $1 \mathrm{~g}$ vs glucosamine [70] $1.5 \mathrm{~g}$ vs NR-INF-02 + glucosamine vs

Avocado-Soybean Unsaponifiables (piascledine) ${ }^{b}$

Blotman 1997 RDBP: ASU ( $n=83)$ vs placebo $(n=80)$ [82]

Maheu et al. RDBP: piascledine $300 \mathrm{mg}(n=85)$ vs 1998 [81] placebo $(n=79)$ (knee and hip OA)

Lequesne et al. RBDP: piascledine $300 \mathrm{mg}(n=55)$ vs 2002 [95] placebo $(n=53)$

Pavelka et al. RDB: piascledine $300 \mathrm{mg}$ vs

2010 [96] chondroitin $1200 \mathrm{mg}(n=263)$

Maheu et al. RDBP: piascledine $300 \mathrm{mg}(n=345)$ 2014 [97]

Parameters measured

results

Thai modified WOMAC, 6 min walk and patient satisfaction

Treadmill walking test, WOMAC and Karnofsky, and oxidative stress levels, inflammatory markers.

Treadmill walking test, WOMAC and Karnofsky scales

stiffness score

VAS AND Knee Injury and

Osteoarthritis Outcome Score

Serum Coll-2-1, Coll-2-INO2, Fib3-1, Fib3-2, CRP, MPO, CTX-II. VAS pain

Pain

COX 2 levels in sinovial fluid at time $=$ All improved no difference between groups 0 and at 4 weeks

VAS and WOMAC

Lequesne's, Initially all groups received NSAID

Lequesne's, VAS for pain, and NSAID

JSW, VAS pain, global assessment

WOMAC, Lequesne's, VAS, global assessment, use of rescue medication

JSW, WOMAC, Lequesne's
All improved in both groups $(p<.001)$ with no intergroup differences

8 months - all measures improved with Meriva $(p<0.05)$

4 month - similar improvements in both groups. Use of NSAIDS decreased in both groups

6 weeks - improvement in WOMAC, VAS, Lequesne's pain scores $(p<.0010)$ but not in stiffness score

No difference between groups for pain and function.

6 months - Coll-2-1 decreased. No change in pain

6 months - improved in all $(p<.0001)$, use of other treatments decreased $(p<0001)$

6 weeks - all treatments showed significant improvement over baseline and placebo

6 months - Lequesne's improved and NSAID use

decreased

$<0.001)$, pain $(p<0.003)$ and NSAID usage.

2 years - no statistical difference in JSW or clinica parameters.

6 months +2 month follow up. All parameters improved during treatment. Stabilized or improved in the follow up. No differences between groups.

3 years - fewer progressors in the ASU group. No differences in clinical measurements.
Trial only 4 weeks

Safety profile better for curcumin

Not a blind study

Not a blind study

Mild gastrointestinal symptoms reported in both groups

Small group size, submaximal doses The effects of two treatments is not additive

Not a blind study, no control

Not a blind study

Significant improvements started in 6 weeks

Short trial, no report of change in clinical symptoms

Small study (<30/ group) and over shor time period. NR-INF-02 is curcuminoid free extract of $C$. longa

Over time pain similar in both groups

Improvement more marked in hip OA. Posthoc analysis - when OA was severe, ASU slowed the disease progression

Statistical significance not achieved due to large variability. 
Table 1 Knee OA antioxidant supplements based on turmeric, avocado and Boswellia (Continued)

Boswellia serrata

Sengupta 2008 RDBP: 5-Loxin (B. serrata extract

[85] enriched with $30 \%$ AKBA) $(n=75)$

Sengupta et al. RDBP: Aflapin $100 \mathrm{mg}$ vs 5-Loxin

2010 [86] $\quad 100 \mathrm{mg}$ vs placebo $(n=20 /$ group $)$

Gupta et al. Open: Shallaki tablet $(6 \mathrm{~g} / \mathrm{d})$ or tablet 2011 [98] and ointment together ( $n=56$ total)

Vishal et al. RDBP: Alfapin vs placebo $(n=30 /$

$2011[88]$ group)
Knee pain, inflection, walking distance
frequency of swelling, radiology

Pain and function VAS, WOMAC and

Lequesne's

WOMAC, Lequesne's, VAS, and serum 90 days - improvement in pain and physical function in biochemical, hematological and urine both treatment groups. changes

Pain, stiffness and swelling, mental state (Jung scales). Radiology, hematology and biochemistry

WOMAC, Lequesne's and VAS for pain 30 days - significant improvement in pain and function and serum biochemical,

hematological and urine changes

Kulkarni et al., RDBP crossover: Articulin-F capsule (W. Pain, stiffness, grip strength, Ritchie

$1991[76$

somnifera, $B$ serrata $C$ longa Zinc com- articular index disability score,

plex) $(n=42)$

Chopra et al. RDBP: RA-11 (W. somnifera, B. serrata,

2004 [99]

Z. officinale, and C. longa) vs placebo radiology.

WOMAC, VAS and hematological,

urine and biochemical tests $(n=90)$

Chopra et al. RDBP: Glucosamine vs celecoxib vs $2013[89,100] \quad$ SGCG ${ }^{C}$ vs SGC $(n=440)$
Weight bearing pain modified WOMAC
Small group size

in all parameters except radiology

90 days - improvement in stiffness, function and pain

2 months - both groups reported significant improvements in pain, stiffness and swelling and by radiology

but not in biochemistry

3 months 2 weeks washout crossover 3 months - pain and Small prospective study. disability were significantly improved over placebo.

32 week - WOMAC, VAS improved over placebo

Very high dropout rate

24 weeks - improvement in pain and function in all

Some patients had adverse hepatic

groups. For WOMAC pain, SGCG worked marginally better effects of SGCG and SGC.

than SGC.

${ }^{a}$ The trials types were random single blind (RSB), random double blind with or without placebo (RDBP, RDB) or open

${ }^{b}$ Studies supported by Laboratoires Expanscience, Courbevoie, France)

'SGCG capsule (400 mg) contained Zingiber officinale, Tinospora cordifolia, Phyllanthus emblica and B. serrata. The SGC capsule (400 mg) was similar to SGCG (both for content and quantity) except for the absence of $B$.

serrata extract and a higher quantity of other ingredients 
Biocurcumax increased the oral bioavailability of curcumin when compared to curcumin alone or curcumin plus lecithin. However, there are no reports using this preparation for OA.

Studies on the management of knee OA have also been conducted using Curcuma extracts in combination with other substances (Table 1). One trial used the combination of roots of Withania somnifera, the stem of Boswellia serrata and rhizomes of Curcuma longa and a zinc complex. There was a significant improvement in pain relief and function [76]. Ainat - a preparation containing devil's claw, turmeric and bromelain also showed a clinically relevant improvement in acute and chronic pain [77].

\section{Avocado-soya extract}

Avocado and soybean oils are used for manufacturing soap and the unsaponifiable fraction from these oils is termed avocado/soybean unsaponifiable (ASU). ASU has been tested in the management of OA. ASU contains phytosterols, $\beta$-sitosterol, campesterol, and stigmasterol, fat soluble vitamins, triterpene fatty acids and possibly furan fatty acids, but the identity of the active components in it is unknown [78]. Several formulations of ASU are available on the market as supplements. Piascledine, which contains the unsaponifiables as one part from avocado and two from soybean, is a unique patented preparation [79]. In articular chondrocyte cultures ASU may modulate NFk $\beta$ levels to inhibit inflammatory cytokines and stimulate collagen synthesis [78]. Animal studies also support its benefits in OA.

A PubMed search in August 2015 with the words "avocado/ASU" and "osteoarthritis" and "knee" showed 25 entries of which five were human clinical trials on pain and function of OA in hip and knee (Table 1). Literature on the human trials of ASU in hip and knee OA, and for the rationale of this therapy has been reviewed recently [78]. It is pointed out that there may be a conflict of interest in these studies. Also, one should consider that soybean protein alone may be beneficial for $\mathrm{OA}$ and this complicates the interpretation of the benefits of ASU [80].

The effect of ASU on patients with hip $(n=50)$ or knee $(n=114)$ OA was examined in a randomised, double blind, placebo controlled, multicenter trial with a 6 month treatment period (Table 1) [81]. After the 6 months its efficacy was greater than that of the placebo $(P<0.001$ for intergroup difference at month 6$)$. The decrease in the Lequesne index (from $9.7 \pm 0.3$ to $6.8 \pm 0.4$ ) was significantly greater in the ASU group than in the placebo group (from $9.4 \pm 0.3$ to $8.9 \pm 0.4$ ). Fewer patients treated with ASU required NSAIDs (48\%) than those in the placebo group (63\%) ( $p=$ 0.054). The improvements appeared to be more marked in patients with hip than those with knee OA. Another similar study confirmed these findings [82]. In yet another study, the effects of two doses (300 or $600 \mathrm{mg}$ daily) of ASU were compared in patients with knee OA over 3 months (Table 1) [83]. Both doses were effective. At day 90, NSAIDs and analgesics intake decreased to less than half in $71 \%$ patients receiving ASU (300 mg or $600 \mathrm{mg}$ ) compared to $36 \%$ in the placebo group $(p<0.01)$. The Lequesne's index dropped by 3.9, 2.9 and 1.9 points with $600 \mathrm{mg}$ ASU, $300 \mathrm{mg}$ ASU and placebo, respectively $(p<0.01)$.

\section{Boswellia}

Resins from trees of Boswellia serrata, and other species of this genus, have been used for arthritis and other diseases in Ayurvedic medicine since ancient times in India where it is termed shallaki or salai (http://en.wikipedia.org/wiki/Boswellia_serrata). In Europe, it is known as olibanum or Frankincense which is mentioned several times in the Bible (http://www.threemagi.com/frankincense.html). Boswellia resins contain several different boswellic acids such as beta-boswellic acid, keto-betaboswellic acid, and acetyl-keto-beta-boswellic acid (AKBA). AKBA is an inhibitor of the lipoxygenase pathway and is suggested to have anti-inflammatory properties [84].

A PubMed search in August 2015 with the words "Boswellia/shallaki/salai/AKBA" and "osteoarthritis" and "knee" showed 18 entries of which only nine were clinical trials, on Boswellia alone or in combination with other substances, with different diagnostic measurements, durations, controls, blindness and conflicts of interests. This included several proprietary preparations from Boswellia extracts (Table 1). Most studies examined mainly pain relief or improvements in function. The Boswellia extract preparations aflapin and 5-loxin were both shown to be beneficial in arthritis although the studies used small numbers of patients, were conducted over short periods only, and did not meet all rigorous criteria [85-88]. Nevertheless, almost all the studies have shown some benefit for OA (Table 1). In some studies, preparations combinations of Boswellia and several other substances were used (Table 1). A small random double blind crossover study conducted in 1991 found that articulin (combination of W. somnifera $450 \mathrm{mg}$, B. serrata $100 \mathrm{mg}$, C. longa $50 \mathrm{mg}$, Zinc complex $50 \mathrm{mg}$ ) improved pain and functional symptoms of OA (Table 1) [76]. However, a literature search showed that the study was not repeated with a larger size. A more extensive study compared two drugs termed SGCG and SGC, both containing several herbs but only SGCG containing Boswellia (Table 1) [89]. Both drugs were effective against OA symptoms but the drug containing Boswellia was marginally more effective in reducing the WOMAC pain symptoms. 


\section{Ayurvedic and Chinese medicines}

Several herbal products containing antioxidants are used in Ayurvedic medicine. For example, roots of Withania somniferum are used to prepare ashwagandha. It contains withanaloids, most importantly withaferin A [90]. Tinospora cordifolia (Guduchi) is considered a divine herb and it contains diterpenoids termed tinosporides [91]. Emblica officinalis (or Phyllanthus emblica) is termed amla or amlaki and is rich in vitamin $C$ and emblicanins A and B [92]. Zingiber officinale (Ginger) root is not only used extensively in cooking but also has several medicinal antioxidants. The analysis of volatile oils of fresh and dried ginger showed camphene, $p$-cineole, $\alpha$-terpineol, zingiberene and pentadecanoic acid as major components [93]. There are several studies demonstrating the usefulness of these antioxidants in OA. A major complication in comparing their usefulness comes from a lack of an exact parallelism between the pathological classifications in Ayurvedic system versus the Western system of medicine. Further, several studies have used only small populations, have fewer controls and their methods of analysis may lend to bias. The characteristics of the studies are presented in a recent review along with their risk of bias according to Cochrane risk of bias tool [38]. Their study included 19 randomized and 14 non-randomized controlled trials on 12 different drugs and three non-pharmaceutical interventions. They concluded that "The drugs Rumalaya and Shunti-Guduchi seem to be safe and effective drugs for treatment of OA-patients, based on these data. However, several limitations relate to clinical research on Ayurveda. Well-planned, well-conducted and well-published trials are warranted to improve the evidence for Ayurvedic interventions." However, part of the problem is that these are very complex proprietary preparations with undisclosed exact compositions. For example, Rumalaya contains Mahayograj guggul, Shankha bhasama, purified Shilajeet, Swarnamakshik bhasama, Latakasthuri and Shallaki, Jaya galangal, Licorice and Tinospora Gulancha. Chemical compositions of each of these is not exactly known.

Chinese herbal medicines have been used for treating $\mathrm{OA}$ in China for centuries. The most commonly prescribed OA medicines in Taiwan are Du-huo-ji-shengtang plus Shen-tong-zhu-yu-tang, and the triple-drug combination was Du-huo-ji-sheng-tang, Gu-sui-pu (Drynaria fortune (Kunze) J. Sm.), and Xu-Duan (Himalaya teasel) [37]. These are all polyplant preparations which have not been critically evaluated as per today's criteria. One study examined the preparation Duhuo Jisheng Tang for treating OA of the knee [94]. This preparation contains parts from 15 different herbs and its chemical constituents include coumarins, phytosterols, polysaccharides, flavanoids, monoterpene glycoside, galloyl glucoses, saponins, ginsenosides and alkaloids. Patients ( $n=68$ ) of both genders and $59.2 \pm 10.2$ years of age were given a dose $2.5 \mathrm{~g}$ twice daily for 4 weeks. There was a highly significant improvement in the WOMAC scores (pain, stiffness and function) and in visual analog scale for pain. As noted, the study used a small number of subjects for a short duration and there was no placebo group.

\section{Synopsis}

OA is a disease of inflammation of large articulate joints with the tibiofemoral joint inflammation being more prevalent. There is no known cure for this disease but considerable progress has been made in joint replacement surgery. The pharmacological strategies for coping with OA include glucosamine plus chondroitin sulfate, hyaluronic acid or corticosteroid injections and NSAIDs.

The pathophysiology of OA is consistent with the hypothesis that the inflammatory molecules and ROS increase with the onset of the disease. Therefore, it is logical to think that antioxidant supplements may be of some benefit. Curcumin which is isolated from Curcuma longa may be efficacious for pain relief and function retention in OA patients. Several products which increase oral bioavailability of curcumin may be promising. Another beneficial product may be ASU. Boswellia resins may also be beneficial and proprietary preparations derived from it and containing AKBA may also have good efficacy. All of these products have fewer adverse effects than the chronic use of NSAIDs. Ayurvedic and ancient Chinese medicines may also be beneficial.

\section{Critical appraisal}

The problem of defining OA has been elegantly discussed in a Clinical Viewpoint [2]. Common understanding today is that OA is a sequential process: loss of proteoglycans on the cartilage surface, death of chondrocytes in deeper layers, hypertrophy and aggregation of remaining chondrocytes, formation of surface cracks parallel to articular face, flow of synovial fluids into these defects, immune reactions due to cartilage fractures, penetration of synovial fluid into defects and cyst formation, osteophyte formation leading to joint crepitus and stiffness, restriction of movements and narrowing of intra-articular space. Pain severity increases with the various stages. One of the problems is that radiographic imaging may show joint space narrowing without any loss in range in motion or any other symptoms. Radiographic imaging does not show cartilage damage and hence MRI, ultrasound, and optical coherence tomography have been introduced recently for diagnosis. However, most studies in drug trials restrict to methods such as WOMAC, VAS or Lequesne index using questionnaires or walking or stair climbing. Each of these indices 
contains a large number of items which deter from a focus on the definition of the disease. With the exception of quantitative measures such as distance walked in $6 \mathrm{~min}$, the methods are highly subjective and a source of bias due to their subjectivity. Stair climbing is a good functional measure but it may often depend on cardiorespiratory abilities in addition to osteoarthritis.

A major hurdle in today's evidence based medicine is money. When money is to be made such as in proprietary or patented drugs, a large investment is expected in the trials. However, when a traditional supplement is to be tested, financial investments may be small and hence large trials do not occur. Conflicts of interest - declared or undeclared may also come about due to such investments. For example, turmeric has been used in Ayurvedic and Chinese medicine for arthritis since ancient times. Curcumin was isolated from it in 1815 [61]. Curcumin has been tested extensively for its benefits in arthritis and other diseases. It is interesting that the bioavailability of this compound is extremely low when given orally in powdered forms in capsules. Yet, when consumed in large doses, it is beneficial for OA. Commercial proprietary preparations using curcumin have been developed to increase its bioavailability. It is ironic that one of the products is made from reconstituting curcumin with curcumin free components from turmeric. Another interesting product contains curcumin and piperine which can be isolated from black pepper. It enhances the bioavailability of curcumin. These products are being tested for their efficacies. The Indian housewife uses turmeric and black pepper routinely in cooking. Yet, the efficacy of this product will not be tested since there is nothing proprietary in it. Even if money were available, taste and colour of turmeric and black pepper would deter a randomized double blind clinical study. Placebos would also be hard to find. This example reflects the type of difficulty one has in evaluating the usefulness of the literature in this area. These difficulties often muddle the clarity and usefulness of the conclusions.

\section{Recommendations}

This review shows that there is some evidence for benefits of antioxidant supplements in pain relief and function in knee OA. These supplements with the most evidence include curcumin, avocado-soya bean unsaponifiables, Boswellia and several preparations used in Ayurvedic and Chinese medicine. These should be tested further and used, at least, to decrease the use of NSAIDs which have more adverse effects.

Ancient medicine should be tested as such rather than proprietary products made from them. It should be assessed whether diet with turmeric and black pepper results in sufficient levels of serum curcumin. Promotion of dietary habits may be more economical and of longer term benefit than the development of products made from extracts used in ancient medicine.

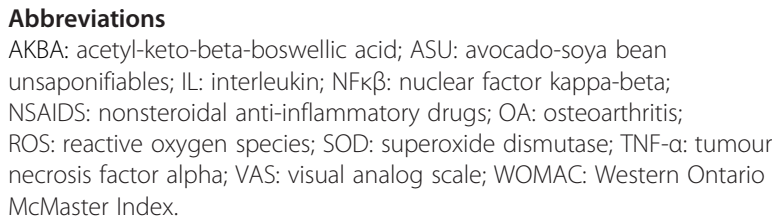

The authors declare that they have no competing interests.

\section{Authors' contributions}

AKG was the planner and the main author in this work. SES did most of the literature survey, assisted in making Table 1 and editing the manuscript. All authors read and approved the final manuscript.

\section{Acknowledgements}

The authors thank Dr. Eva Werstiuk for pre-reviewing this manuscript. This work was supported only from internal funds from McMaster University. There is no conflict of interest due to any funding from nutraceutical or pharmaceutical companies.

Received: 28 August 2015 Accepted: 21 December 2015

Published online: 05 January 2016

\section{References}

1. Alexander CJ. Utilisation of joint movement range in arboreal primates compared with human subjects: an evolutionary frame for primary osteoarthritis. Ann Rheum Dis. 1994;53:720-5.

2. Wade GJ. Rethinking the model of osteoarthritis: a clinical viewpoint. J Am Osteopath Assoc. 2011;111:631-7.

3. Yuan XL, Meng HY, Wang YC, Peng J, Guo QY, Wang AY, et al. Bonecartilage interface crosstalk in osteoarthritis: potential pathways and future therapeutic strategies. Osteoarthr Cartil. 2014;22:1077-89.

4. Ashford S, Williard J. Osteoarthritis: a review. Nurse Pract. 2014;39:1-8.

5. Wojdasiewicz P, Poniatowski LA, Szukiewicz D. The role of inflammatory and anti-inflammatory cytokines in the pathogenesis of osteoarthritis. Mediators Inflamm. 2014;2014:561459.

6. Burr DB, Gallant MA. Bone remodelling in osteoarthritis. Nat Rev Rheumatol. 2012;8:665-73.

7. Henrotin Y, Pesesse L, Sanchez C. Subchondral bone and osteoarthritis: biological and cellular aspects. Osteoporos Int. 2012;23 Suppl 8:S847-51.

8. Zhang LZ, Zheng HA, Jiang Y, Tu YH, Jiang PH, Yang AL. Mechanical and biologic link between cartilage and subchondral bone in osteoarthritis. Arthritis Care Res (Hoboken). 2012;64:960-7.

9. Kim KK, Won Y, Kim TG, Baek MH, Choi J. Comparison of the chemical composition of subchondral trabecular bone of medial femoral condyle between with advanced osteoarthritis and without osteoarthritis. J Bone Metab. 2015:22:93-7.

10. Saklatvala J. Tumour necrosis factor alpha stimulates resorption and inhibits synthesis of proteoglycan in cartilage. Nature. 1986;322:547-9.

11. Seguin CA, Bernier SM. TNFalpha suppresses link protein and type II collagen expression in chondrocytes: role of MEK1/2 and NF-kappaB signaling pathways. J Cell Physiol. 2003;197:356-69.

12. Poree B, Kypriotou M, Chadjichristos $C$, Beauchef $G$, Renard E, Legendre F, et al. Interleukin-6 (IL-6) and/or soluble IL-6 receptor down-regulation of human type $\|$ collagen gene expression in articular chondrocytes requires a decrease of Sp1.Sp3 ratio and of the binding activity of both factors to the COL2A1 promoter. J Biol Chem. 2008;283:4850-65.

13. Sun JM, Sun LZ, Liu J, Su BH, Shi L. Serum interleukin-15 levels are associated with severity of pain in patients with knee osteoarthritis. Dis Markers. 2013;35:203-6.

14. Scanzello CR, Umoh E, Pessler F, Diaz-Torne C, Miles T, Dicarlo E, et al. Local cytokine profiles in knee osteoarthritis: elevated synovial fluid interleukin-15 differentiates early from end-stage disease. Osteoarthr Cartil. 2009;17:1040-8.

15. Chen B, Deng Y, Tan Y, Qin J, Chen LB. Association between severity of knee osteoarthritis and serum and synovial fluid interleukin 17 concentrations. J Int Med Res. 2014;42:138-44. 
16. Juhl C, Christensen R, Roos EM, Zhang W, Lund H. Impact of exercise type and dose on pain and disability in knee osteoarthritis: a systematic review and meta-regression analysis of randomized controlled trials. Arthritis Rheumatol. 2014;66:622-36.

17. Bellamy N, Buchanan WW, Goldsmith CH, Campbell J, Stitt LW. Validation study of WOMAC: a health status instrument for measuring clinically important patient relevant outcomes to antirheumatic drug therapy in patients with osteoarthritis of the hip or knee. J Rheumatol. 1988;15:1833-40.

18. Braun HJ, Gold GE. Diagnosis of osteoarthritis: imaging. Bone. 2012;51:278-88.

19. Schiphof D, Oei EH, Hofman A, Waarsing JH, Weinans H, Bierma-Zeinstra SM. Sensitivity and associations with pain and body weight of an MRI definition of knee osteoarthritis compared with radiographic Kellgren and Lawrence criteria: a population-based study in middle-aged females. Osteoarthr Cartil. 2014;22:440-6.

20. Foster NE, Healey EL, Holden MA, Nicholls E, Whitehurst DG, Jowett S, et al. A multicentre, pragmatic, parallel group, randomised controlled trial to compare the clinical and cost-effectiveness of three physiotherapy-led exercise interventions for knee osteoarthritis in older adults: the BEEP trial protocol (ISRCTN: 93634563). BMC Musculoskelet Disord. 2014;15:254.

21. Golightly YM, Allen KD, Caine DJ. A comprehensive review of the effectiveness of different exercise programs for patients with osteoarthritis. Phys Sportsmed. 2012:40:52-65.

22. Gur H, Cakin N, Akova B, Okay E, Kucukoglu S. Concentric versus combined concentric-eccentric isokinetic training: effects on functional capacity and symptoms in patients with osteoarthrosis of the knee. Arch Phys Med Rehabil. 2002;83:308-16.

23. Page CJ, Hinman RS, Bennell KL. Physiotherapy management of knee osteoarthritis. Int J Rheum Dis. 2011;14:145-51.

24. Salli A, Sahin $H$, Baskent A, Ugurlu $H$. The effect of two exercise programs on various functional outcome measures in patients with osteoarthritis of the knee: a randomized controlled clinical trial. Isokinet Exerc Sci. 2010;18:201-9.

25. Messier SP, Loeser RF, Miller GD, Morgan TM, Rejeski WJ, Sevick MA, et al. Exercise and dietary weight loss in overweight and obese older adults with knee osteoarthritis: the Arthritis, Diet, and Activity Promotion Trial. Arthritis Rheum. 2004;50:1501-10.

26. Hay EM, Foster NE, Thomas E, Peat G, Phelan M, Yates HE, et al. Effectiveness of community physiotherapy and enhanced pharmacy review for knee pain in people aged over 55 presenting to primary care: pragmatic randomised trial. BMJ. 2006;333:995

27. Adatia A, Rainsford KD, Kean WF. Osteoarthritis of the knee and hip. Part II: therapy with ibuprofen and a review of clinical trials. J Pharm Pharmacol. 2012;64:626-36

28. McCarberg B, Tenzer P. Complexities in the pharmacologic management of osteoarthritis pain. Curr Med Res Opin. 2013:29:539-48.

29. Frestedt $J$, Kuskowski MA, Zenk JL. A natural seaweed derived mineral supplement (Aquamin F) for knee osteoarthritis: a randomised, placebo controlled pilot study. Nutr J. 2009:8:7.

30. Miller MJ, Mehta K, Kunte S, Raut V, Gala J, Dhumale R, et al. Early relief of osteoarthritis symptoms with a natural mineral supplement and a herbomineral combination: a randomized controlled trial [ISRCTN38432711]. J Inflamm (Lond). 2005;2:11.

31. Nieman DC, Shanely RA, Luo B, Dew D, Meaney MP, Sha W. A commercialized dietary supplement alleviates joint pain in community adults: a double-blind, placebo-controlled community trial. Nutr J. 2013;12:154.

32. Kanzaki N, Saito K, Maeda A, Kitagawa Y, Kiso Y, Watanabe K, et al. Effect of a dietary supplement containing glucosamine hydrochloride, chondroitin sulfate and quercetin glycosides on symptomatic knee osteoarthritis: a randomized, double-blind, placebo-controlled study. J Sci Food Agric. 2012:92:862-9.

33. Martel-Pelletier J, Roubille C, Abram F, Hochberg MC, Dorais M, Delorme $P$, et al. First-line analysis of the effects of treatment on progression of structural changes in knee osteoarthritis over 24 months: data from the osteoarthritis initiative progression cohort. Ann Rheum Dis. 2013;74:547-56.

34. Matsuno H, Nakamura H, Katayama K, Hayashi S, Kano S, Yudoh K, et al. Effects of an oral administration of glucosamine-chondroitin-quercetin glucoside on the synovial fluid properties in patients with osteoarthritis and rheumatoid arthritis. Biosci Biotechnol Biochem. 2009;73:288-92.

35. Nakasone $Y$, Watabe K, Watanabe K, Tomonaga A, Nagaoka I, Yamamoto T, et al. Effect of a glucosamine-based combination supplement containing chondroitin sulfate and antioxidant micronutrients in subjects with symptomatic knee osteoarthritis: a pilot study. Exp Ther Med. 2011;2:893-9.
36. Reginster JY, Deroisy R, Rovati LC, Lee RL, Lejeune E, Bruyere O, et al. Longterm effects of glucosamine sulphate on osteoarthritis progression: a randomised, placebo-controlled clinical trial. Lancet. 2001;357:251-6.

37. Chen FP, Chang CM, Hwang SJ, Chen YC, Chen FJ. Chinese herbal prescriptions for osteoarthritis in Taiwan: analysis of National Health Insurance dataset. BMC Complement Altern Med. 2014;14:91.

38. Kessler CS, Pinders L, Michalsen A, Cramer H. Ayurvedic interventions for osteoarthritis: a systematic review and meta-analysis. Rheumatol Int. 2015; 35:211-32.

39. Grover AK, Samson SE. Antioxidants and vision health: facts and fiction. Mol Cell Biochem. 2014;388:173-83.

40. Abdali D, Samson SE, Grover AK. How effective are antioxidant supplements in obesity and diabetes? Med Princ Pract. 2015;24(3):201-15.

41. Walia M, Kwan CY, Grover AK. Effects of free radicals on coronary artery. Med Princ Pract. 2003:12:1-9.

42. Fridovich I. Oxygen: how do we stand it? Med Princ Pract. 2013;22:131-7.

43. Liochev SI. Free radicals: how do we stand them? Anaerobic and aerobic free radical (chain) reactions involved in the use of fluorogenic probes and in biological systems. Med Princ Pract. 2014;23:195-203.

44. Altindag O, Erel O, Aksoy N, Selek S, Celik H, Karaoglanoglu M. Increased oxidative stress and its relation with collagen metabolism in knee osteoarthritis. Rheumatol Int. 2007;27:339-44.

45. Sutipornpalangkul W, Morales NP, Charoencholvanich K, Harnroongroj T. Lipid peroxidation, glutathione, vitamin E, and antioxidant enzymes in synovial fluid from patients with osteoarthritis. Int J Rheum Dis. 2009;12:324-8.

46. Regan EA, Bowler RP, Crapo JD. Joint fluid antioxidants are decreased in osteoarthritic joints compared to joints with macroscopically intact cartilage and subacute injury. Osteoarthr Cartil. 2008;16:515-21.

47. Forman HJ, Davies KJ, Ursini F. How do nutritional antioxidants really work: nucleophilic tone and para-hormesis versus free radical scavenging in vivo. Free Radic Biol Med. 2014;66:24-35.

48. Jones DP. Redefining oxidative stress. Antioxid Redox Signal. 2006;8:1865-79.

49. Morquette B, Shi Q, Lavigne P, Ranger P, Fernandes JC, Benderdour M. Production of lipid peroxidation products in osteoarthritic tissues: new evidence linking 4-hydroxynonenal to cartilage degradation. Arthritis Rheum. 2006:54:271-81.

50. Ziskoven C, Jager M, Kircher J, Patzer T, Bloch W, Brixius K, et al. Physiology and pathophysiology of nitrosative and oxidative stress in osteoarthritic joint destruction. Can J Physiol Pharmacol. 2011;89:455-66.

51. Shen CL, Smith BJ, Lo DF, Chyu MC, Dunn DM, Chen CH, et al. Dietary polyphenols and mechanisms of osteoarthritis. J Nutr Biochem. 2012;23:1367-77.

52. McAlindon TE, Jacques $P$, Zhang $Y$, Hannan MT, Aliabadi $P$, Weissman $B$, et al. Do antioxidant micronutrients protect against the development and progression of knee osteoarthritis? Arthritis Rheum. 1996;39:648-56.

53. McAlindon T, LaValley M, Schneider E, Nuite M, Lee JY, Price LL, et al. Effect of vitamin D supplementation on progression of knee pain and cartilage volume loss in patients with symptomatic osteoarthritis: a randomized controlled trial. JAMA. 2013;309:155-62.

54. Jordan JM, De Roos AJ, Renner JB, Luta G, Cohen A, Craft N, et al. A casecontrol study of serum tocopherol levels and the alpha- to gammatocopherol ratio in radiographic knee osteoarthritis: the Johnston County Osteoarthritis Project. Am J Epidemiol. 2004;159:968-77.

55. Wang $Y$, Prentice LF, Vitetta $L$, Wluka AE, Cicuttini FM. The effect of nutritional supplements on osteoarthritis. Altern Med Rev. 2004;9:275-96.

56. Williams FM, Skinner J, Spector TD, Cassidy A, Clark IM, Davidson RM, et al. Dietary garlic and hip osteoarthritis: evidence of a protective effect and putative mechanism of action. BMC Musculoskelet Disord. 2010;11:280.

57. Wang $Y$, Hodge AM, Wluka AE, English DR, Giles GG, O'Sullivan $R$, et al. Effect of antioxidants on knee cartilage and bone in healthy, middle-aged subjects: a cross-sectional study. Arthritis Res Ther. 2007:9:R66.

58. Schumacher HR, Pullman-Mooar S, Gupta SR, Dinnella JE, Kim R, McHugh MP. Randomized double-blind crossover study of the efficacy of a tart cherry juice blend in treatment of osteoarthritis (OA) of the knee. Osteoarthr Cartil. 2013;21:1035-41.

59. Farid R, Rezaieyazdi Z, Mirfeizi Z, Hatef MR, Mirheidari M, Mansouri H, et al. Oral intake of purple passion fruit peel extract reduces pain and stiffness and improves physical function in adult patients with knee osteoarthritis. Nutr Res. 2010;30:601-6.

60. Singh $G$, Kapoor IP, Singh P, de Heluani CS, de Lampasona MP, Catalan CA. Comparative study of chemical composition and antioxidant activity of 
fresh and dry rhizomes of turmeric (Curcuma longa Linn.). Food Chem Toxicol. 2010;48:1026-31.

61. Gupta SC, Patchva S, Koh W, Aggarwal BB. Discovery of curcumin, a component of golden spice, and its miraculous biological activities. Clin Exp Pharmacol Physiol. 2012;39:283-99.

62. Kertia N, Asdie AH, Rochmah W, Marsetyawan. Ability of curcuminoid compared to diclofenac sodium in reducing the secretion of cycloxygenase-2 enzyme by synovial fluid's monocytes of patients with osteoarthritis. Acta Med Indones. 2012;44:105-13.

63. Kuptniratsaikul V, Thanakhumtorn S, Chinswangwatanakul P, Wattanamongkonsil L, Thamlikitkul V. Efficacy and safety of Curcuma domestica extracts in patients with knee osteoarthritis. J Altern Complement Med. 2009;15:891-7.

64. Kuptniratsaikul V, Dajpratham P, Taechaarpornkul W, Buntragulpoontawee M, Lukkanapichonchut P, Chootip C, et al. Efficacy and safety of Curcuma domestica extracts compared with ibuprofen in patients with knee osteoarthritis: a multicenter study. Clin Interv Aging. 2014;9:451-8.

65. Pinsornsak P, Niempoog S. The efficacy of Curcuma Longa L. extract as an adjuvant therapy in primary knee osteoarthritis: a randomized control trial. J Med Assoc Thai. 2012;95 Suppl 1:S51-8.

66. Belcaro G, Dugall M, Luzzi R, Ledda A, Pellegrini L, Cesarone MR, et al. Meriva(R)+Glucosamine versus Condroitin+Glucosamine in patients with knee osteoarthritis: an observational study. Eur Rev Med Pharmacol Sci. 2014;18:3959-63.

67. Henrotin Y, Gharbi M, Dierckxsens Y, Priem F, Marty M, Seidel L, et al. Decrease of a specific biomarker of collagen degradation in osteoarthritis, Coll2-1, by treatment with highly bioavailable curcumin during an exploratory clinical trial. BMC Complement Altern Med. 2014;14:159.

68. Panahi Y, Rahimnia AR, Sharafi M, Alishiri G, Saburi A, Sahebkar A. Curcuminoid treatment for knee osteoarthritis: a randomized double-blind placebo-controlled trial. Phytother Res. 2014;28:1625-31.

69. Shoba G, Joy D, Joseph T, Majeed M, Rajendran R, Srinivas PS. Influence of piperine on the pharmacokinetics of curcumin in animals and human volunteers. Planta Med. 1998;64:353-6.

70. Madhu K, Chanda K, Saji MJ. Safety and efficacy of Curcuma longa extract in the treatment of painful knee osteoarthritis: a randomized placebocontrolled trial. Inflammopharmacology. 2013;21:129-36.

71. Belcaro G, Cesarone MR, Dugall M, Pellegrini L, Ledda A, Grossi MG, et al. Efficacy and safety of Meriva(R), a curcumin-phosphatidylcholine complex during extended administration in osteoarthritis patients. Altern Med Rev. 2010;15:337-44.

72. Appelboom T, Maes N, Albert A. A new curcuma extract (flexofytol(R)) in osteoarthritis: results from a belgian real-life experience. Open Rheumatol J. 2014:8:77-81.

73. Storka A, Vcelar B, Klickovic U, Gouya G, Weisshaar S, Aschauer S, et al. Safety, tolerability and pharmacokinetics of liposomal curcumin in healthy humans. Int J Clin Pharmacol Ther. 2015;53:54-65.

74. Ying $X$, Chen $X$, Cheng $S$, Shen $Y$, Peng L, Xu HZ. Piperine inhibits IL-beta induced expression of inflammatory mediators in human osteoarthritis chondrocyte. Int Immunopharmacol. 2013;17:293-9.

75. Antony B, Merina B, Iyer VS, Judy N, Lennertz K, Joyal S. A pilot cross-over study to evaluate human oral bioavailability of BCM-95CG (Biocurcumax), a novel bioenhanced preparation of curcumin. Indian J Pharm Sci. 2008;70:445-9.

76. Kulkarni RR, Patki PS, Jog VP, Gandage SG, Patwardhan B. Treatment of osteoarthritis with a herbomineral formulation: a double-blind, placebocontrolled, cross-over study. J Ethnopharmacol. 1991;33:91-5.

77. Conrozier T, Mathieu P, Bonjean M, Marc JF, Renevier JL, Balblanc JC. A complex of three natural anti-inflammatory agents provides relief of osteoarthritis pain. Altern Ther Health Med. 2014:20 Suppl 1:32-7.

78. Christiansen BA, Bhatti S, Goudarzi R, Emami S. Management of Osteoarthritis with Avocado/Soybean Unsaponifiables. Cartilage. 2015;6:30-44.

79. Ragle RL, Sawitzke AD. Nutraceuticals in the management of osteoarthritis : a critical review. Drugs Aging. 2012;29:717-31.

80. Arjmandi BH, Khalil DA, Lucas EA, Smith BJ, Sinichi N, Hodges SB, et al. Soy protein may alleviate osteoarthritis symptoms. Phytomedicine. 2004;11:567-75.

81. Maheu E, Mazieres B, Valat JP, Loyau G, Le Loët X, Bourgeois P, et al. Symptomatic efficacy of avocado/soybean unsaponifiables in the treatment of osteoarthritis of the knee and hip: a prospective, randomized, doubleblind, placebo-controlled, multicenter clinical trial with a six-month treatment period and a two-month followup demonstrating a persistent effect. Arthritis Rheum. 1998;41:81-91.
82. Blotman F, Maheu E, Wulwik A, Caspard H, Lopez A. Efficacy and safety of avocado/soybean unsaponifiables in the treatment of symptomatic osteoarthritis of the knee and hip. A prospective, multicenter, three-month, randomized, double-blind, placebo-controlled trial. Rev Rhum Engl Ed. 1997; 64:825-34.

83. Appelboom T, Schuermans J, Verbruggen G, Henrotin Y, Reginster JY. Symptoms modifying effect of avocado/soybean unsaponifiables (ASU) in knee osteoarthritis. A double blind, prospective, placebo-controlled study. Scand J Rheumatol. 2001;30:242-7.

84. Kimmatkar N, Thawani V, Hingorani L, Khiyani R. Efficacy and tolerability of Boswellia serrata extract in treatment of osteoarthritis of knee-a randomized double blind placebo controlled trial. Phytomedicine. 2003;10:3-7.

85. Sengupta K, Alluri KV, Satish AR, Mishra S, Golakoti T, Sarma KV, et al. A double blind, randomized, placebo controlled study of the efficacy and safety of 5-Loxin for treatment of osteoarthritis of the knee. Arthritis Res Ther. 2008;10:R85

86. Sengupta K, Krishnaraju AV, Vishal AA, Mishra A, Trimurtulu G, Sarma KV, et al. Comparative efficacy and tolerability of 5-Loxin and AflapinAgainst osteoarthritis of the knee: a double blind, randomized, placebo controlled clinical study. Int J Med Sci. 2010;7:366-77.

87. Sengupta K, Kolla JN, Krishnaraju AV, Yalamanchili N, Rao CV, Golakoti T, et al. Cellular and molecular mechanisms of anti-inflammatory effect of Aflapin: a novel Boswellia serrata extract. Mol Cell Biochem. 2011;354:189-97.

88. Vishal AA, Mishra A, Raychaudhuri SP. A double blind, randomized, placebo controlled clinical study evaluates the early efficacy of aflapin in subjects with osteoarthritis of knee. Int J Med Sci. 2011:8:615-22.

89. Chopra A, Saluja M, Tillu G, Sarmukkaddam S, Venugopalan A, Narsimulu G, et al. Ayurvedic medicine offers a good alternative to glucosamine and celecoxib in the treatment of symptomatic knee osteoarthritis: a randomized, double-blind, controlled equivalence drug trial. Rheumatology (Oxford). 2013;52:1408-17.

90. Vanden Berghe W, Sabbe L, Kaileh M, Haegeman G, Heyninck K. Molecular insight in the multifunctional activities of Withaferin A. Biochem Pharmacol. 2012;84:1282-91.

91. Huang C, Li W, Ma F, Li Q, Asada Y, Koike K. Tinospinosides D, E, and tinospin $E$, further clerodane diterpenoids from Tinospora sagittata. Chem Pharm Bull (Tokyo). 2012;60:1324-8.

92. Pozharitskaya ON, Ivanova SA, Shikov AN, Makarov VG. Separation and evaluation of free radical-scavenging activity of phenol components of Emblica officinalis extract by using an HPTLC-DPPH* method. J Sep Sci. 2007:30:1250-4

93. El Ghorab AH, Nauman M, Anjum FM, Hussain S, Nadeem M. A comparative study on chemical composition and antioxidant activity of ginger (Zingiber officinale) and cumin (Cuminum cyminum). J Agric Food Chem. 2010;58:8231-7.

94. Lai JN, Chen HJ, Chen CC, Lin JH, Hwang JS, Wang JD. Duhuo jisheng tang for treating osteoarthritis of the knee: a prospective clinical observation. Chin Med. 2007;2:4.

95. Lequesne M, Maheu E, Cadet C, Dreiser RL. Structural effect of avocado/ soybean unsaponifiables on joint space loss in osteoarthritis of the hip. Arthritis Rheum. 2002:47:50-8.

96. Pavelka K, Coste P, Geher P, Krejci G. Efficacy and safety of piascledine 300 versus chondroitin sulfate in a 6 months treatment plus 2 months observation in patients with osteoarthritis of the knee. Clin Rheumatol. 2010;29:659-70.

97. Maheu E, Cadet C, Marty M, Moyse D, Kerloch I, Coste P, et al. Randomised, controlled trial of avocado-soybean unsaponifiable (Piascledine) effect on structure modification in hip osteoarthritis: the ERADIAS study. Ann Rheum Dis. 2014;73:376-84.

98. Gupta PK, Samarakoon SM, Chandola HM, Ravishankar B. Clinical evaluation of Boswellia serrata (Shallaki) resin in the management of Sandhivata (osteoarthritis). Ayu. 2011;32:478-82.

99. Chopra A, Lavin P, Patwardhan B, Chitre D. A 32-week randomized, placebocontrolled clinical evaluation of RA-11, an Ayurvedic drug, on osteoarthritis of the knees. J Clin Rheumatol. 2004:10:236-45.

100. Chopra A, Saluja M, Tillu G, Venugopalan A, Narsimulu G, Sarmukaddam S, et al. Evaluating higher doses of Shunthi - Guduchi formulations for safety in treatment of osteoarthritis knees: a Government of India NMITLI arthritis project. J Ayurveda Integr Med. 2012;3:38-44. 